\title{
Artificial neural network approach for estimation of surface specific humidity and air temperature using Multifrequency Scanning Microwave Radiometer
}

\author{
Randhir Singh, B G Vasudevan, P K Pal and P C Joshi \\ Meteorology and Oceanography Group, Space Applications Centre (ISRO), Ahmedabad 380 015, India.
}

\begin{abstract}
Microwave sensor MSMR (Multifrequency Scanning Microwave Radiometer) data onboard Oceansat-1 was used for retrieval of monthly averages of near surface specific humidity $\left(Q_{a}\right)$ and air temperature $\left(T_{a}\right)$ by means of Artificial Neural Network (ANN). The MSMR measures the microwave radiances in 8 channels at frequencies of $6.6,10.7,18$ and $21 \mathrm{GHz}$ for both vertical and horizontal polarizations.

The artificial neural networks (ANN) technique is employed to find the transfer function relating the input MSMR observed brightness temperatures and output $\left(Q_{a}\right.$ and $\left.T_{a}\right)$ parameters. Input data consist of nearly 28 months (June 1999 - September 2001) of monthly averages of MSMR observed brightness temperature and surface marine observations of $Q_{a}$ and $T_{a}$ from Comprehensive OceanAtmosphere Data Set (COADS).

The performance of the algorithm is assessed with independent surface marine observations. The results indicate that the combination of MSMR observed brightness temperatures as input parameters provides reasonable estimates of monthly averaged surface parameters. The global root mean square (rms) differences are $1.0^{\circ} \mathrm{C}$ and $1.1 \mathrm{~g} \mathrm{~kg}^{-1}$ for air temperature and surface specific humidity respectively.
\end{abstract}

\section{Introduction}

Surface latent heat flux (LHF), is one of the dominant components in the surface energy balance and plays a key role in both the hydrological cycle and in coupling the ocean and atmosphere (Peixoto and Oort 1992). In the past it has been necessary to rely exclusively on the in situ observations to estimate this important parameter. However, with the advent of satellite technology, there are unique and complementary ways to remotely derive surface heat fluxes. Satellite observations have high spatial and temporal resolution and hence are best suited for studying the large-scale phenomenon. The surface latent heat flux can be computed using bulk formula (Schulz et al 1997) in which the SST, wind speed, near surface specific humidity $\left(Q_{a}\right)$ and air temperature $\left(T_{a}\right)$ must be known. The principal issue with the satellite method (bulk formula), however, is that accurate estimations of near surface specific humidity $\left(Q_{a}\right)$ and air temperature $\left(T_{a}\right)$ are not directly available from remote sensing measurements.

India has launched several remote sensing satellites under the series of IRS satellites. IRS-P4 satellite is the fourth satellite launched by the Indian launch vehicle PSLV from Shriharikota, India. This satellite is also known as Oceansat-1. Both the names are interchangeably used for this satellite. This satellite carries two oceanographic play-loads, an ocean color monitor (OCM) and a multi-frequency scanning microwave radiometer (MSMR). The multi-frequency scanning microwave radiometer onboard IRS P4 has the capability of global coverage with two-day repetivity. MSMR is an eight band (four frequency in dual polariza-

Keywords. ANN; COADS; MSMR; SSM/I; NCEP. 
tions) passive microwave radiometer with a conical scan mechanism. MSMR provides measurements of brightness temperature at 6.6, 10.7, 18, and $21 \mathrm{GHz}$ frequencies in both horizontal and vertical polarizations. The brightness temperature of the ocean is a function of ocean temperature and emissivity. The emissivity depends upon the sea surface temperature, wind speed and also on sensors parameters (frequency, polarization and viewing angle). When measured from space, the ocean brightness temperature is modified by transmission through atmosphere, whose primary variables of importance to surface microwave observations are water vapour, cloud liquid water and rainfall. In order to determine sea surface temperature, corrections are necessary for these variables. The microwave absorption spectrum of water vapour has a resonance at $22 \mathrm{GHz}$. Thus, measurements at or near this frequency (e.g., $21 \mathrm{GHz}$ for MSMR) are sensitive to variation in total atmospheric water vapour. The operational geophysical parameters available from MSMR measurements are wind speed, cloud liquid water, integrated water vapour and sea surface temperature over global oceans. For retrieval of geophysical parameters from MSMR brightness temperature, a variety of theoretical approaches have been utilized. These include the radiative transfer simulation of a variety of atmospheres and then followed by development of retrieval algorithms (Gohil et al 2000).

Use of vertically integrated water vapour content $(W)$ as a predictor is a common way to determine the near surface specific humidity from satellite measurements. The correlation between both these quantities depends heavily on the time scale considered. The method of Liu (1986) (hereafter L86) determines the monthly marine surface-layer humidity with a simple polynomial regression of $Q_{a}$ versus $W$ (total precipitable water). This simple formula can be used with any retrieval algorithm which determines $W$. Unfortunately, errors in $Q_{a}$ originating from the $Q_{a}-W$ relation can result in large errors in latent heat flux. This was shown by Esbensen et al (1993), who compared one year of LHF derived from satellite data using the $Q_{a}-W$ relation with in situ estimates from COADS observations. He showed systematic error of over $2 \mathrm{~g} / \mathrm{kg}$ in the satellite estimates (L86) of monthly averages $Q_{a}$.

Since air temperature $\left(T_{a}\right)$ is key to climate studies, the search for improved approaches to determine it from space is an active research topic. Few attempts (Jourdan and Gautier 1995; Konda et al 1996) have been made on the estimation of near surface air temperature $\left(T_{a}\right)$ from satellite data. A very simple method is to assume slightly unstable conditions (Schulz et al 1997) at any location at any time and set $T_{a}=\mathrm{SST}-1$, where $\mathrm{SST}$ is sea surface temperature. Another simple method is to compute $T_{a}$ from the retrieved $Q_{a}$ assuming a constant relative humidity, e.g., 80\% (Liu 1988), or using climatological relative humidity. This might be accurate enough to compute exchange coefficients but seems to be too rough to determine sensible heat flux.

Therefore, it is clear from the above discussion that, though progress has been made in the estimation of $Q_{a}$ and $T_{a}$ yet some methods need to be explored in order to make suitable flux computation from satellite data more accurate. There has been an increasing interest in recent years in the use of artificial neural networks (ANN) as applied to satellite remote sensing retrievals (Jung et al 1998). The ANN algorithms have two salient and attractive features, ANN algorithms are not only computationally efficient, but they are also very useful in representing nonlinear relationships among a set of parameters. Recently Jones et al (1999) tried to invert monthly mean $T_{a}$ from SSM/I (Special Sensor Microwave Imager) measurements of total precipitable water, $W$, and sea surface temperature, SST, analysis from NCEP (National Centre for Environmental Prediction) using neural network techniques. The global rms was stated to be $0.72^{\circ} \pm 0.38^{\circ} \mathrm{C}$.

Motivated by the present status of the satellite retrieval of $Q_{a}$ and $T_{a}$, this paper describes ANN methodology to determine monthly averages of $Q_{a}$ and $T_{a}$ from MSMR measured brightness temperatures of eight channels, $T_{B} 6 \mathrm{v}, T_{B} 6 \mathrm{~h}, T_{B} 10 \mathrm{v}, T_{B} 10 \mathrm{~h}$, $T_{B} 18 \mathrm{v}, T_{B} 18 \mathrm{~h}, T_{B} 21 \mathrm{v}$, and $T_{B} 21 \mathrm{~h}$.

\section{Materials used}

The algorithm developed in this study used monthly mean averages of MSMR brightness temperatures to determine $Q_{a}$ and $T_{a}$. The data record spans the 28 months from June 1999 through September 2001, and is available on a global grid of $1.5^{\circ}$ latitude by $1.5^{\circ}$ longitude resolutions.

In order to develop and evaluate the new methodology, $Q_{a}$ and $T_{a}$ observations from ship reports of Comprehensive Ocean-Atmosphere Data Set (COADS) for the period June 1999 to September 2001 over global oceans were used. As an outcome of joint efforts of several organizations, namely National Oceanic and Atmospheric Administration (NOAA), National Climate Data Centre (NCDC), Cooperative Institute of Research in Environmental Sciences (CIRES), and National Centre for Atmospheric Research (NCAR), the Comprehensive OceanAtmosphere Data Set (COADS) was archived. For the first time, the most efficient and up to date 


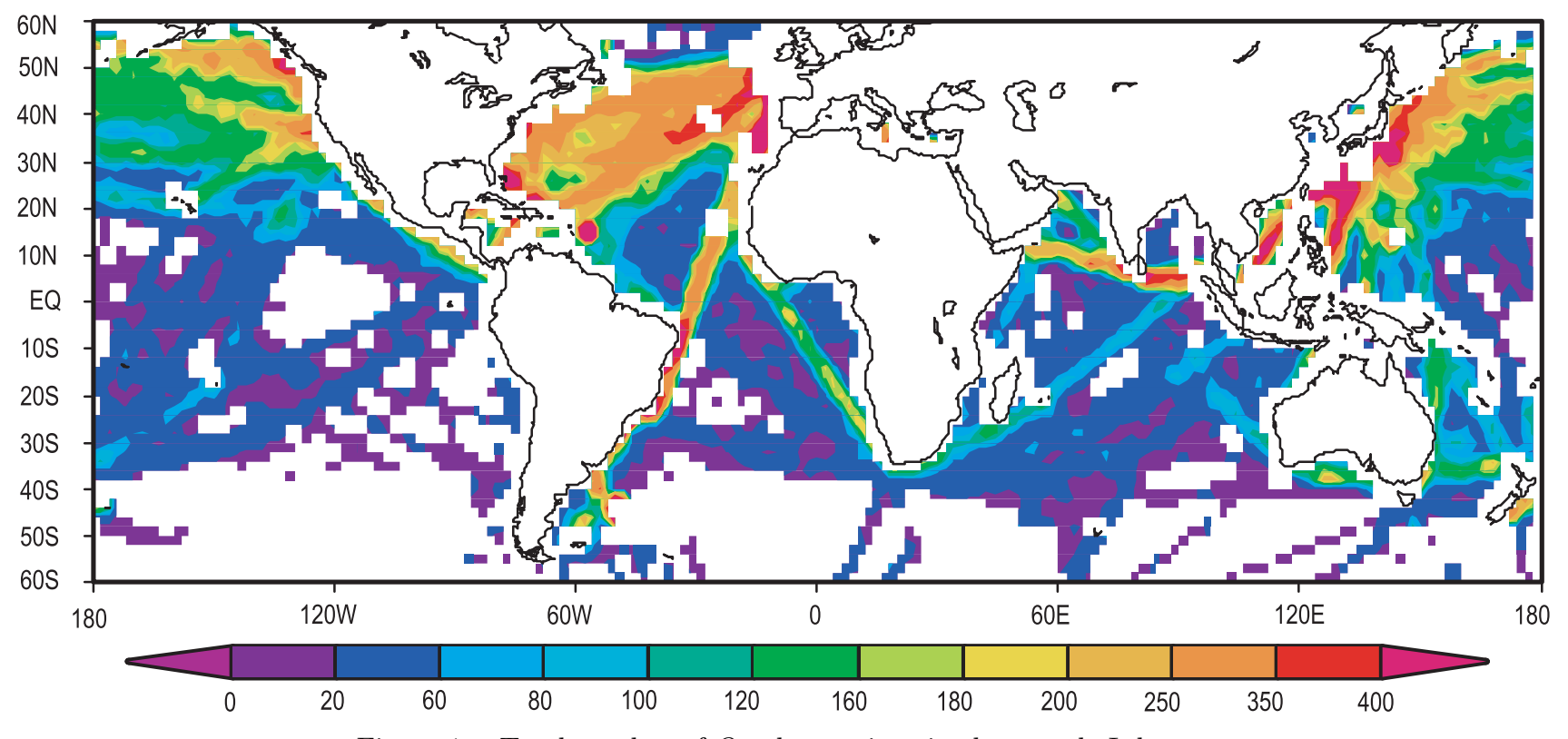

Figure 1. Total number of $Q_{a}$ observations in the sample-I dataset.

techniques have been used to compile all available oceanic and atmospheric data over the world oceans (Fletcher et al 1983; Oort et al 1987). The COADS consists of mean monthly marine surface wind speed, Sea Surface Temperature (SST), near surface specific humidity $\left(Q_{a}\right)$ and near surface air temperature $\left(T_{a}\right)$, compiled and checked for quality. A large number of studies have been carried with this data in order to study the influence of oceans on climate variability (Oort et al 1987; and others).

The collocated datasets of MSMR observed brightness temperatures, $Q_{a}(\mathrm{COADS})$ and $T_{a}$ (COADS) were initially divided into two subsamples. The June 1999 - May 2000 period is designated as sample-I (S-I), and used to train the neural network. Similarly, the June 2000 - September 2001 period was designated as sample-II (S-II) and reserved for evaluating the performance of the methodology (hereafter the ANN method). Figure 1 shows the total number of COADS observations in sample-I (used to train neural network). Maximum number of observations are available in the north Atlantic and Pacific Ocean. One can observe unsampled regions in central Pacific, Indian Ocean and the region beyond $40^{\circ} \mathrm{S}$ is completely unsampled.

\section{Artificial Neural Network (ANN)}

In general, a neural network is a computer model composed of individual processing elements called neurons. The neurons are connected by links in term of weights. Each neuron in one layer has direct connection to the neurons of the subsequent layer.
These layers are referred to as an input layer, hidden layer or an output layer. Generally NN is characterized by its topology, namely, the number of input, output and hidden neurons and how they are interconnected. The multi-layer perceptron (MLP) class of networks consists of multiple layers of computational units, usually interconnected in a feedforward way.

Multi-layer networks use a variety of learning techniques, the most popular being backpropagation (Miller and Emery 1997). It minimizes the error between the target output $\left(Q_{a}, T_{a}\right)$ and the inputs (functions of $T_{B}$ 's) on the data of so called training dataset (S-I) using some predefined error function. By various techniques the error is then fed back through the network. Using this information, the algorithm adjusts the weights of each connection in order to reduce the value of the error-function by some small amount. After repeating this process for a sufficiently large number of training cycles the network will usually converge to some state where the error of the calculations is minimum. Thus one can say that the network has learned a certain target function. To adjust weights properly one applies a general method for nonlinear optimization task such as a gradient descent. For this the derivation of the error-function with respect to the network weights is calculated and the weights are then changed as such that the error decreases (thus going downhill on the surface of the error function). For this reason back-propagation can only be applied on networks with differentiable activation function. The sigmoid, hyperbolic tangent and the gaussian function are the most common choices (Rumelhart et al 1986). For most modeling tasks, the sigmoid function are the base- 
line model to measure results. A general rule of thumb is that the sigmoid will produce the most accurate model; but be slower learning. One advantage is that it has a very simple derivative. Activation functions for the hidden units are needed to introduce nonlinearity into the network. Without nonlinearity, hidden units would not make nets more powerful than just plain perceptrons. It is the nonlinearity (i.e., capability to represent nonlinear functions) that makes multilayer networks so powerful.

\subsection{Training stage and methodology}

An ANN algorithm is applied to derive transfer functions relating the MSMR observed brightness temperature (input) to the ocean surface parameters, near surface specific humidity and air temperature (outputs). The training cycle involved forward feeding of $T_{B}$ 's values in the training set from the input layer to output layer to calculate the $Q_{a}$ and $T_{a}$ mapping errors. MLP is accomplished by iterative adjustment of the weights associated with each connection in the network. The updating algorithm designed for the MLP is based on the back-propagation rule developed by Rumelhart et al (1986), which updates the weights in the network.

A back-propagation network is trained by supervised learning where the sample-I dataset is used. The network is presented with a series of input/output combinations each consisting of an input $T_{B}$ 's and output $Q_{a}$ or $T_{a}$. The target output is the desired response to the given input vector. The output $z$, is to be compared with the desired target value $d$, and their differences $e=d-z$, will be computed. The net function $u_{i j}(x)$ is the weighted sum of the $i$ th input to the $j$ th hidden units:

$$
u_{i j}(x)=\sum_{i=1}^{n} w_{j i} x_{i}=\mathbf{W} \mathbf{x},
$$

where $x_{0}=1, \mathbf{W}$ is the weight matrix, and $\mathbf{x}=$ $\left[1 x_{1} x_{2} \ldots x_{n}\right]^{T}$ is the input vector. Here $n$, is number of input. The output $z_{j}(x)$ is obtained from the $u_{i j}(x)$ via an activation function $\psi(u)$ (sigmoid function):

$$
z_{j}=\psi(u)=\frac{1}{1+e^{-u_{i j}}} .
$$

Given a set of training samples $\{(x(k), d(k)) ; 1 \leq$ $k \leq K\}$, the error back-propagation training begins by feeding all $K$ inputs through the MLP (multi layer perceptron) network and computing the corresponding output $\{z(k) ; 1 \leq k \leq K\}$. The aim of learning is to minimize the instantaneous square error of the output signal computed as:

$$
E=\frac{1}{2} \sum_{k=1}^{K}(d(k)-z(k))^{2}=\frac{1}{2}[e(k)]^{2} .
$$

Adjusting the weight matrix $W$ carries out minimization of $E$. This leads to a nonlinear optimization problem. There are numerous nonlinear optimization algorithms available to solve this problem. The minimization is done by the gradient descent method, where back-propagation involves the chain rule to back-propagate errors from the network's outputs to each of the network's weights (Fausett 1994). Use of gradient based techniques to minimize the error function, needs to solve $d E / d w_{j i}$ for all layers, through repetitive use of the chain rule of differentiation. Finally the weight update at every epoch " $t$ " is given by iterative formula.

$$
w_{i j}(t+1)=w_{i j}(t)+\eta \frac{d E}{d w_{i j}},
$$

where $\eta$ is the learning rate. In the above equation $d E / d w_{j i}$ can be expressed as:

$$
\frac{d E}{d w_{i j}}=-2 \sum_{k=1}^{K} \delta_{j}(k) x_{i}(k),
$$

$\delta_{j}(k)$ is called delta error of $j$ th hidden layer. For a sigmoid activation function the $\delta_{j}(k)$ can be computed as:

$$
\delta_{j}(k)=\frac{\partial E}{\partial u_{i j}}=[d(k)-z(k)] \cdot z(k) \cdot[1-z(k)] .
$$

Each time the weights are updated it is called an epoch (local iteration). Here each input/output combination of the training samples is trained to update the weight until the minimum desired response is obtained. This may require several epochs to attain the minimum error-limit. When the S-I dataset $(1 \leq k \leq K)$ is trained once, then one pass of training is over, many such passes are required to train the $\mathrm{NN}$ for minimization. The term global iteration is the cumulative sum of local iteration over the entire training passes. For each pass the r.m.s is calculated between the desire and the instant retrieved output. Such cases are shown in figures 3(a) and 3(b). In the minimization process, the error limit used was 0.003 with a learning rate of 0.7 . Thus the back-propagation formula computes the delta error from the output layer back towards the input layer, in a layer-bylayer manner. 
Table 1. Evolution of $Q_{a}$ and $T_{a}$, r.m.s error minimization for the training data (sample I) for different $N N$ configuration.

\begin{tabular}{l|cc|cc|cc}
\hline \multirow{2}{*}{$\begin{array}{c}\text { Neural } \\
\text { network }\end{array}$} & \multicolumn{6}{|c}{ Global iterations } \\
\cline { 2 - 7 } $\begin{array}{c}|c| \\
\text { configuration }\end{array}$ & $T_{a}$ & $Q_{a}$ & $T_{a}$ & $Q_{a}$ & $T_{a}$ & $Q_{a}$ \\
\cline { 2 - 7 } $8-8-1$ & 4.00 & 3.36 & 1.56 & 1.63 & 1.14 & 1.40 \\
$8-15-1$ & 3.25 & 3.49 & 1.25 & 1.49 & 1.10 & 1.30 \\
$8-20-1$ & 2.19 & 3.59 & 1.19 & 1.34 & 1.10 & 1.27 \\
\hline $8-8-8-1$ & 3.39 & 3.32 & 1.19 & 1.45 & 1.12 & 1.44 \\
$8-15-15-1$ & 2.96 & 2.02 & 1.19 & 1.56 & 1.12 & 1.22 \\
$8-20-20-1$ & 2.64 & 3.15 & 1.09 & 1.28 & $\mathbf{1 . 0 8}$ & 1.17 \\
\hline $8-8-8-8-1$ & 2.71 & 2.25 & $\mathbf{1 . 0 8}$ & $\mathbf{1 . 1 0}$ & 1.08 & 1.11 \\
$8-15-15-15-1$ & 2.51 & 2.40 & 1.09 & $\mathbf{1 . 1 0}$ & 1.08 & 1.12 \\
$8-20-20-20-1$ & 2.69 & 2.27 & 1.20 & 1.12 & 1.18 & 1.13 \\
\hline
\end{tabular}

In the present study, $Q_{a}$ and $T_{a}$ have been retrieved individually from MSMR data. Different neural network configurations were used to investigate the best configuration yielding minimum r.m.s error. NN with eight neurons in input layer with 1 to 3 hidden layers each containing 8 to 20 neurons and one output neuron for $Q_{a}$ and $T_{a}$ individually has been considered. Variations of r.m.s with the global iterations for such cases are shown in table 1. A large number of epochs are considered and checked for global minimization to prevent over or under training of NN. From table 1 it can be seen that for $T_{a}, 2$ layers-20 neurons per each hidden layer shows minimum r.m.s error after training for about $3 \times 10^{5}$ epochs, and for the $Q_{a}$, 3 layers-15 neurons per each hidden layer shows minimum r.m.s error again after training for about the same epochs, we could select different network configurations for different parameters, In table 1 the MLP with 3-hidden layers each containing 8neurons shows minimum r.m.s error and better convergence for both the parameters $\left(Q_{a}\right.$ and $\left.T_{a}\right)$. Thus the 8-8-8-8-1x architecture has been considered as the final NN model (figure 2), with eight input MSMR $T_{B}$ 's and outputs as either, $Q_{a}$ or $T_{a}$.

The sample-II dataset was used to evaluate the training performance of the $\mathrm{NN}, T_{B}$ 's values were forwarded through NN and the outputs in the output layer were calculated. The r.m.s values were computed on both sample-I and on the sampleII dataset as shown in table 2. An $\mathrm{NN}$ with the minimum r.m.s was selected for the final MSMR algorithms. There is no evidence to conclude that the smallest r.m.s S-I may ensure a better NN performance. The increase in the r.m.s S-I for decrease

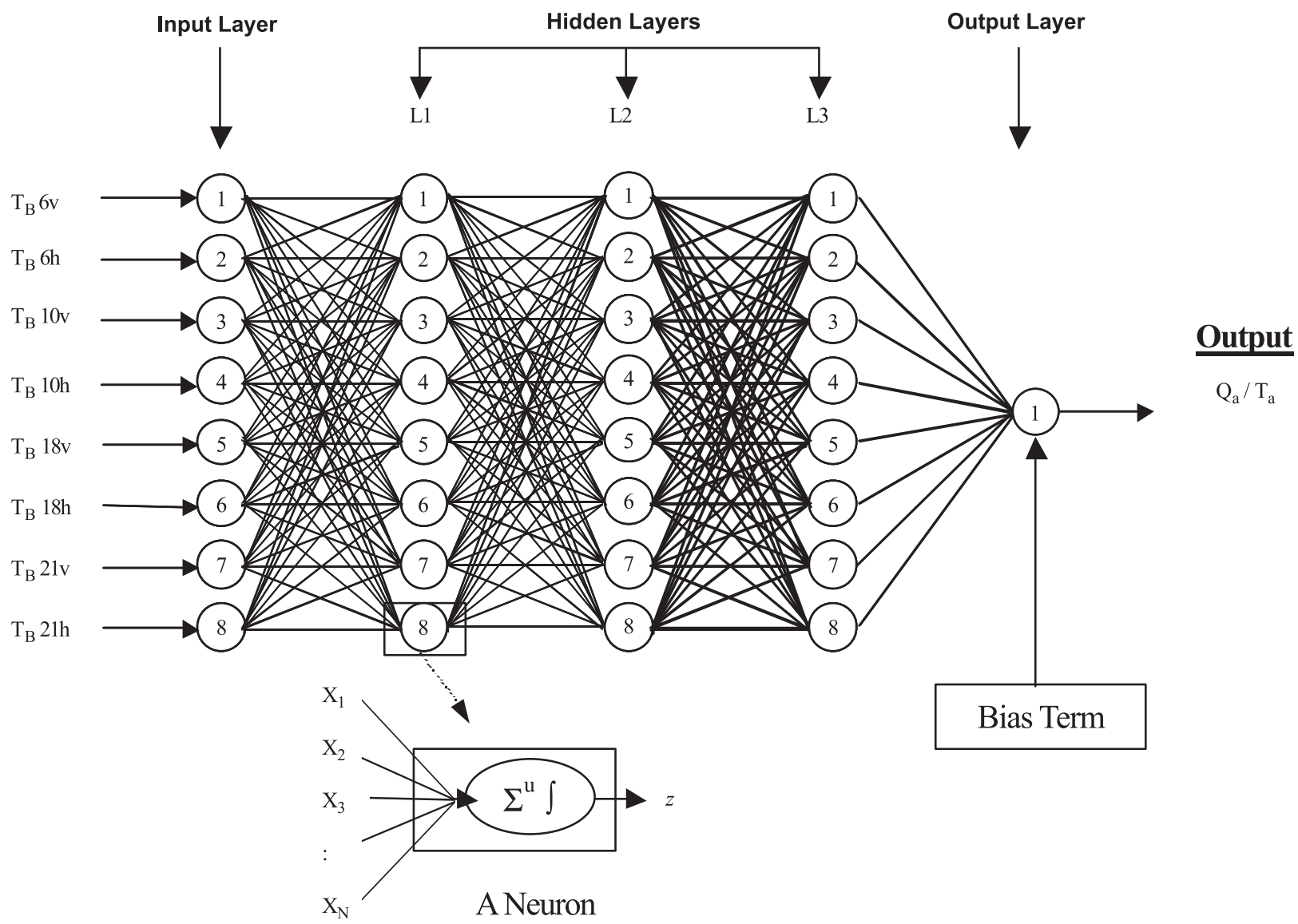

Figure 2. The structure of 5 layers feed-forward neural network and the details of single neuron. 
Table 2. Evolution of $Q_{a}$ and $T_{a}$, r.m.s error on sample $I$ and sample II dataset for $8-8-8-8-1 \times N N$ model.

\begin{tabular}{l|cc|cc|cc}
\hline \multirow{2}{*}{$\begin{array}{c}\text { Neural } \\
\text { network }\end{array}$} & \multicolumn{5}{|c}{ Global iterations } \\
\cline { 2 - 7 } $\begin{array}{c}\text { configuration } \\
\text { 8-8-8-8-1 }\end{array}$ & \multicolumn{2}{|c|}{2000} & \multicolumn{2}{|c}{200000} & \multicolumn{2}{c}{400000} \\
\cline { 2 - 7 } & $T_{a}$ & $Q_{a}$ & $T_{a}$ & $Q_{a}$ & $T_{a}$ & $Q_{a}$ \\
\hline Sample I & 2.71 & 2.25 & $\mathbf{1 . 0 8}$ & $\mathbf{1 . 1 0}$ & 1.08 & 1.11 \\
Sample II & 2.72 & 2.21 & $\mathbf{1 . 0 6}$ & $\mathbf{1 . 0 9}$ & 1.09 & 1.12 \\
\hline
\end{tabular}

r.m.s in r.m.s S-II could show an over training rather than a good training with general patterns for a successful generalization.

The MSMR measured brightness temperatures at 6.6 and $10.7 \mathrm{GHz}$ are sensitive to the SST. Brightness temperatures at 18 and $21 \mathrm{GHz}$ are sensitive to the atmospheric water vapour $(W)$. Air temperature and near surface specific humidity are strongly related (Jones et al 1999) to the sea
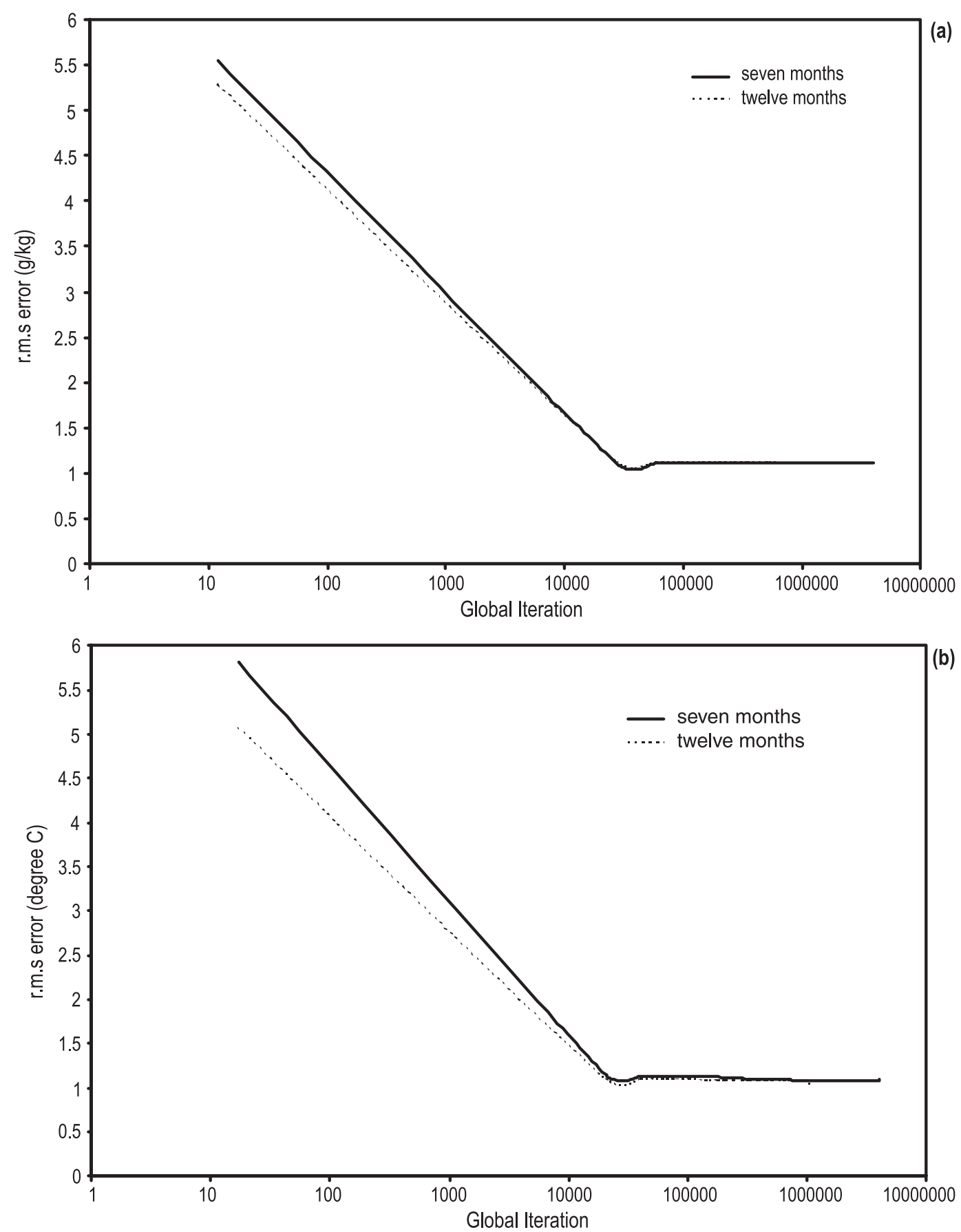

Figure 3. Rms error versus global iteration for 8-8-8-8-1 ANN configuration for two data set in sample-I, (a) humidity, (b) air temperature. 
surface temperature and atmospheric water vapour. Therefore $Q_{a}$ and $T_{a}$ are sensitive to all the brightness temperatures. Hence better performance was achieved when all MSMR brightness temperatures were used.

To see the impact of a number of data points to train the network, two datasets from sample I; seven months and twelve months respectively were selected. Figures ( $3 \mathrm{a}$ and $3 \mathrm{~b}$ ) show the r.m.s error for $Q_{a}$ and $T_{a}$, versus global iteration for these two datasets. In these figures the dotted line corresponds to twelve months and the solid line corresponds to seven months data in sample I. From these figures it is clear that the accuracy is similar in both the cases. If a small data set contains all variability of the parameters the inclusion of more number of data points does not affect the NN performances much. The source code for the algorithm is written in ' $\mathrm{C}$ ', to run under Unix platform with memory requirement of about $256 \mathrm{MB}$.

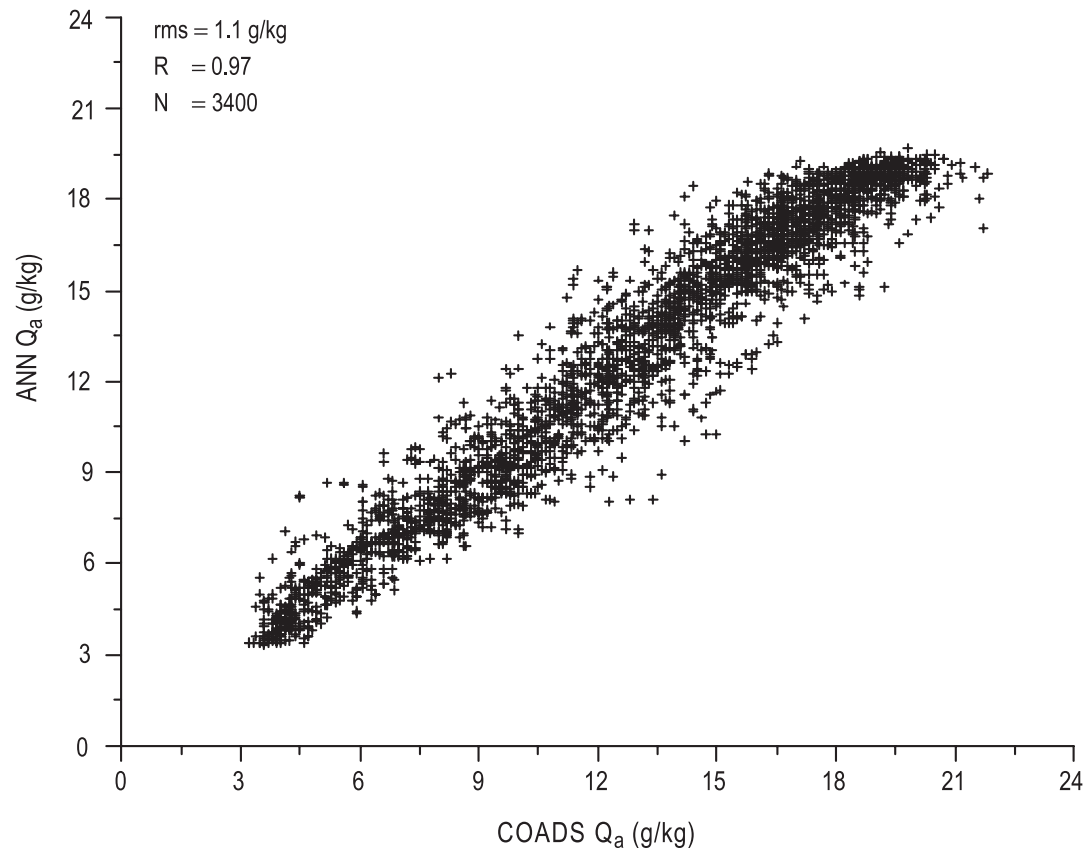

Figure 4. Scatter plot of monthly averaged surface specific humidity from ANN methodology and COADS measurements.

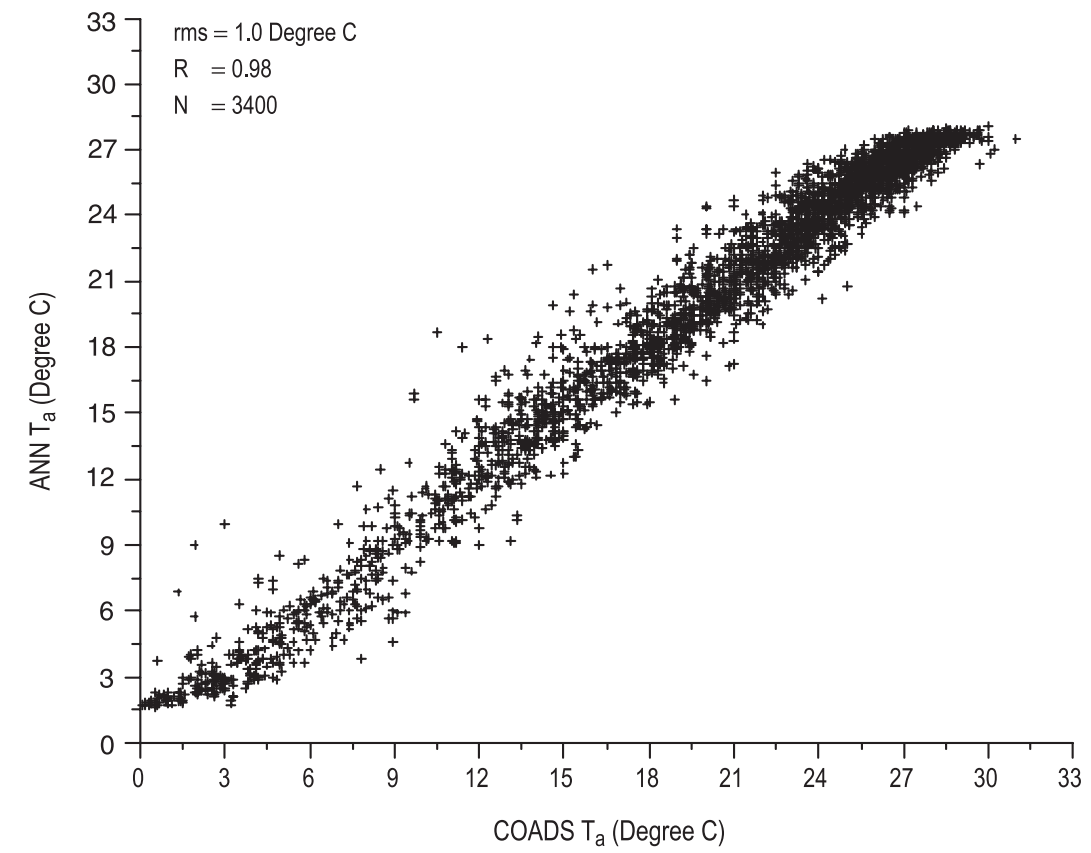

Figure 5. Scatter plot of monthly averaged air temperature from ANN methodology and COADS measurements. 


\section{Results}

The methodology described above was tested by applying the algorithm to the sample II data. Taking COADS as our standard, we compared ANN derived $Q_{a}$ and $T_{a}$ with $Q_{a}$ and $T_{a}$ given in
COADS. Figure 4 shows scatter plot of monthly averages surface specific humidity from COADS versus ANN retrieval. The rms difference between ANN and COADS $Q_{a}$ is $1.1 \mathrm{~g} / \mathrm{kg}$. The ANN retrieved $Q_{a}$ get saturated beyond $20 \mathrm{~g} / \mathrm{kg}$. Thus, the retrieved $Q_{a}$ is underestimated over the warm

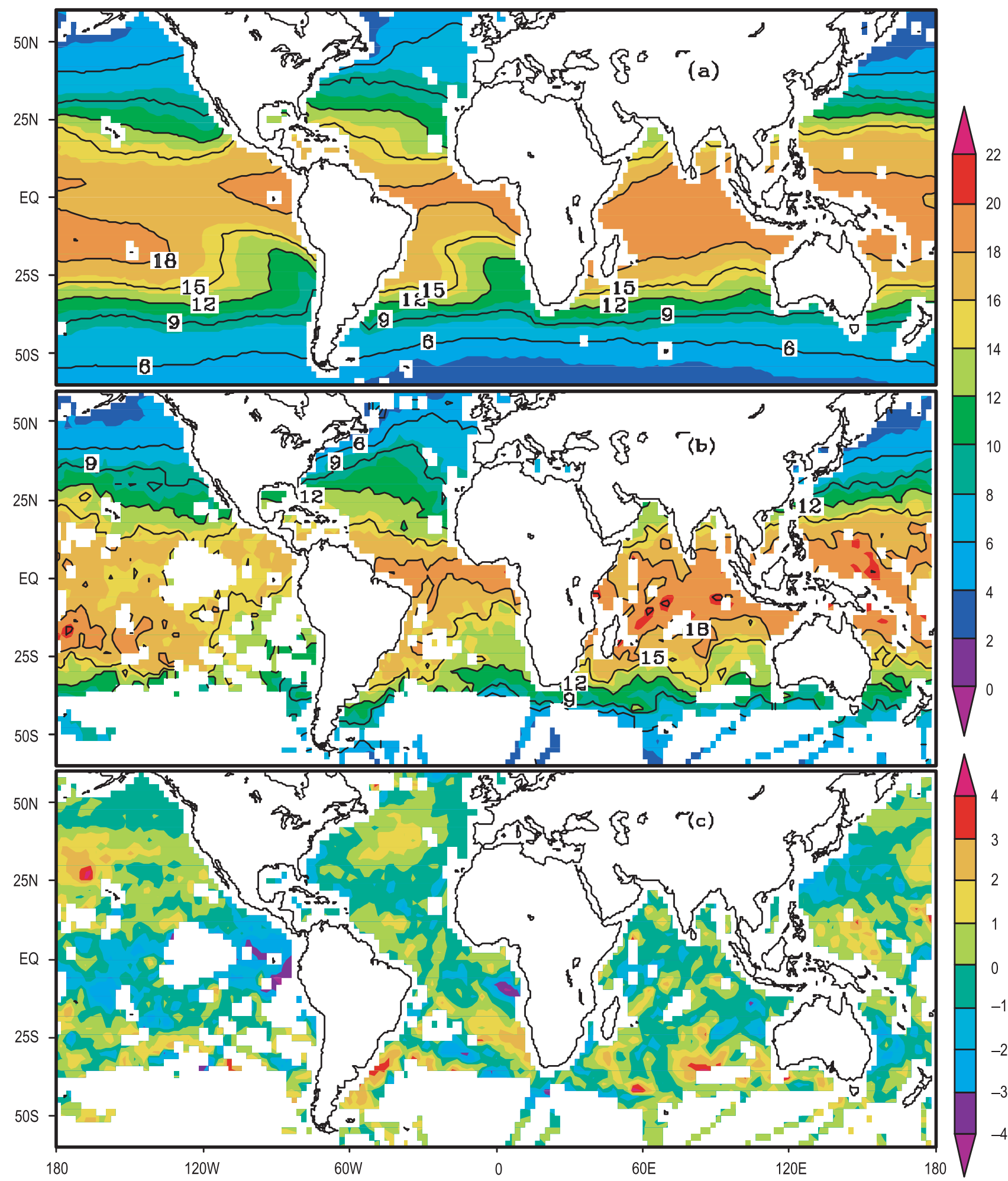

Figure 6. Monthly averaged $Q_{a}(\mathrm{~g} / \mathrm{kg}),(\mathbf{a})$ the ANN retrieved, (b) COADS observed, and (c) the COADS-minus-ANN retrieved, during January. 


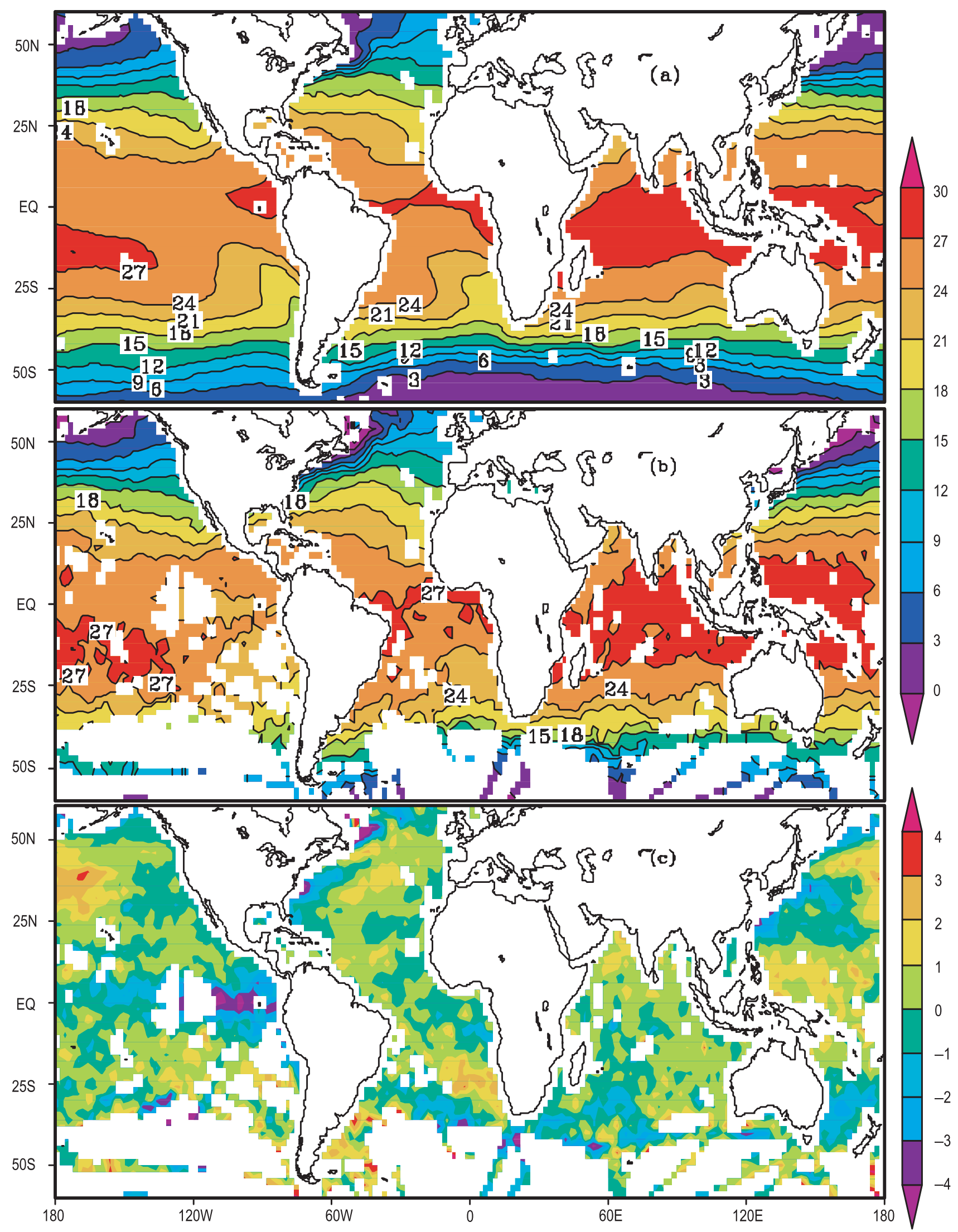

Figure 7. Same as figure 6 but for air temperature $\left({ }^{\circ} \mathrm{C}\right)$. 
pool region (western Pacific and over eastern Indian Ocean). The retrieved $Q_{a}$ overestimates for $Q_{a}<13$, particularly in western boundary current areas (such as the Kuroshio and Gulf Stream) during winter. Figure 5 shows the scatter plot of monthly averages of air temperature from COADS versus the ANN retrieval. The rms difference between ANN and COADS $T_{a}$ is $1.0^{\circ} \mathrm{C}$. The retrieved $T_{a}$ overestimates for $T_{a}<10^{\circ} \mathrm{C}$, but underestimates for $T_{a}>29^{\circ} \mathrm{C}$.

This saturation beyond $20 \mathrm{~g} / \mathrm{kg}$ in $Q_{a}$ and $29^{\circ} \mathrm{C}$ in $T_{a}$ is because of very few values of these orders in the sample I (training data). As we have seen in figure 1, most of the data during the training phase is from north Atlantic and north Pacific Ocean. These oceans do not have such high values of $Q_{a}$ and $T_{a}$.

A better understanding of spatial distribution is given by looking at figures 6 and 7 . In figures 6 and 7, the upper panel (a) is obtained using satellite (ANN retrieved), the middle panel (b) is obtained with COADS data, and the bottom one (c) is COADS-minus-ANN. Figure 6 shows the geographical distribution of $Q_{a}$ during January 2000. The spatial coverage differs strongly between satellite and in situ (COADS) maps, especially in the southern hemisphere, and prevents any comparison between the two- $Q_{a}$ estimates south of $40^{\circ} \mathrm{S}$.

Qualitatively the agreement between the maps is good in the sense that similar structures are found at the same location. In both the cases high humidity was found in the ITCZ region and to the north of the equator, the South Pacific Convergence Zone (SPCZ) running southwest from New Guinea, and the warm pool in the western Pacific and eastern Indian Oceans. The overall pattern in both cases is matching well.

The systematic negative differences (figure 6c) are mainly concentrated in high-evaporation regions during winter. Systematic positive differences are mainly concentrated over south Indian Ocean, central Pacific and south Atlantic Ocean. The differences between these two estimates are within $\pm 0.6 \mathrm{~g} / \mathrm{kg}$ over most of the north Pacific and Atlantic Oceans, although small pockets of differences more than $1 \mathrm{~g} / \mathrm{kg}$ are evident very near to the coast over these oceans. These large differences are due to the land influence on the brightness temperature. Another region of large differences (more than $\pm 1 \mathrm{~g} / \mathrm{kg}$ ) are south Indian and south-central Pacific Ocean. Over these oceans it is very difficult to judge the accuracy of retrieved $Q_{a}$ because the ship observations are very less.

Figure 7 shows the geographical distribution of $T_{a}$ during January 2000. The most important feature of the air temperature distribution is the large east west gradient in the Pacific Ocean. Further there exists the warm pool region in the western Pacific and eastern Indian Ocean. The maximum differences between COADS given temperature and temperature obtained from ANN are in the warm pool region of western Pacific Ocean. The ANN retrievals show less aerial extension of western Pacific Ocean warm pool region compared to COADS. Large differences (figure 7c) between the COADS observed and ANN retrieved air temperature are found in southern Indian Ocean and over south-central Pacific Ocean. The differences are within $\pm 0.5^{\circ}$ over most of the northern Atlantic and Pacific Ocean. Similar to $Q_{a}$, in $T_{a}$ also large differences are observed in southern hemisphere.

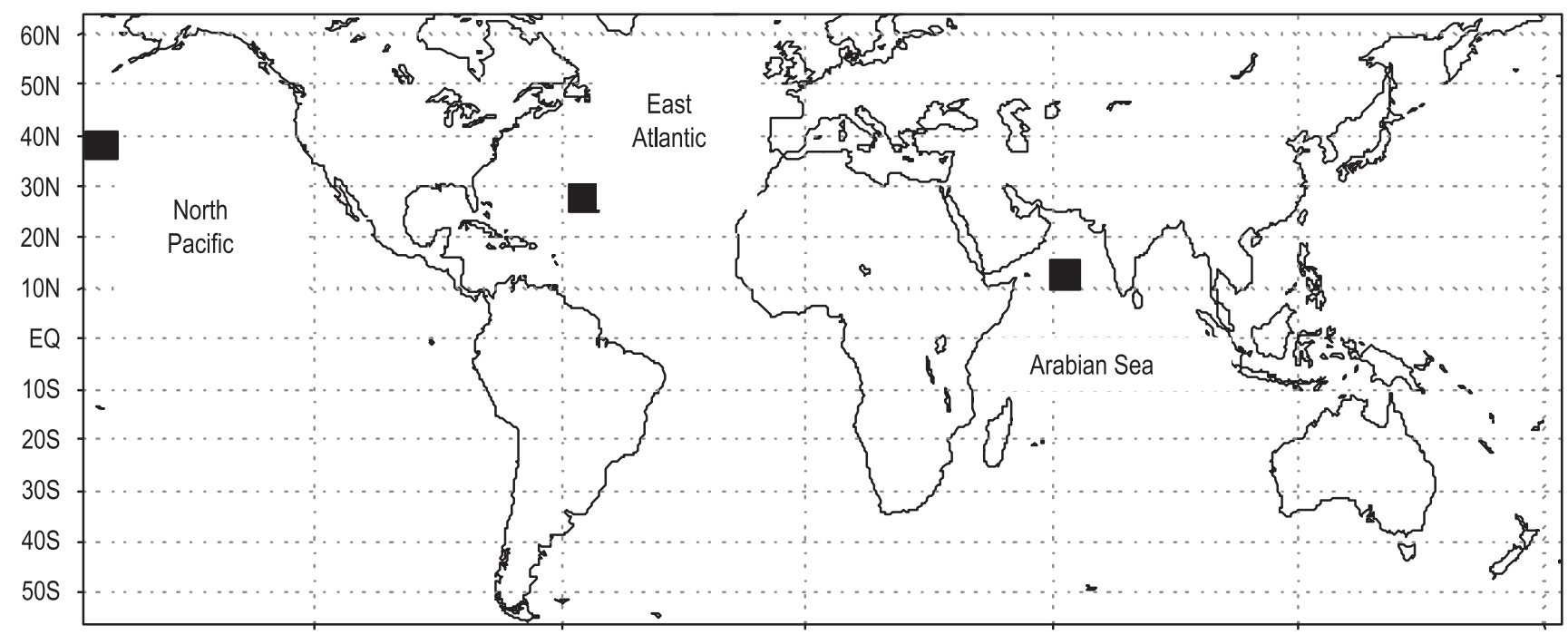

Figure 8. Locations for producing time series for selected regions are shown in black boxes. 

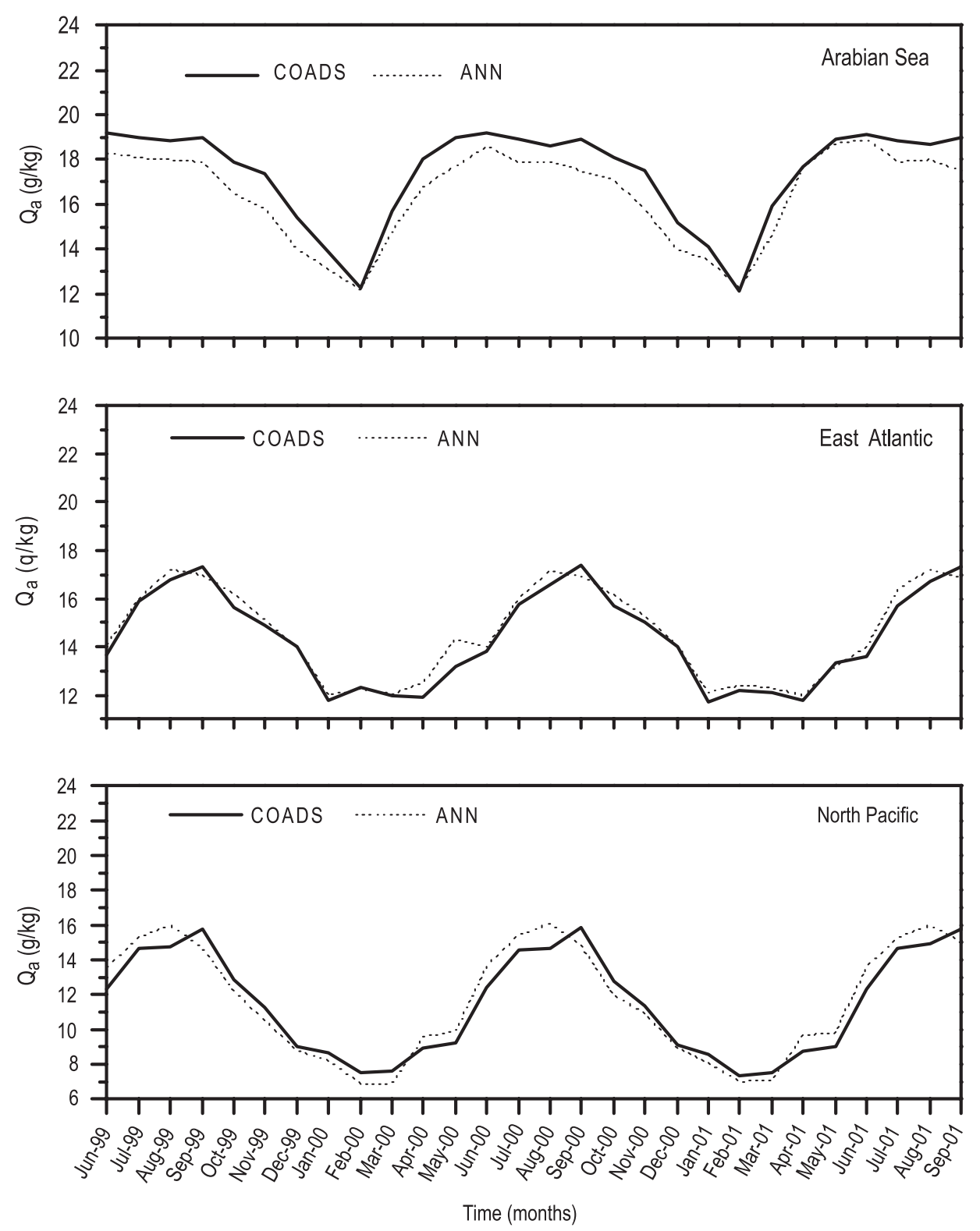

Figure 9. Time series of spatial averages of monthly averages of surface specific humidity $(\mathrm{g} / \mathrm{kg})$ at different regions.

Finally the ANN algorithm was applied to the entire record of MSMR observed brightness temperature (June 1999 - September 2001) in order to achieve a broader prospective of the time variability during the data record. Figure 8 shows the domain used to get spatial averages to produce time series at different locations. Figure 9 shows the time series of $Q_{a}$ averaged over different regions (shown in figure 8). Good agreement over the whole period is observed between ANN method (dotted line) and COADS (solid line) over the entire region. Likewise, the time series of $T_{a}$ (figure 10) shows good agreement between COADS and ANN retrieval.

\section{Conclusions}

Near surface specific humidity $\left(Q_{a}\right)$ and air temperature $\left(T_{a}\right)$ are important parameters for the calculation of surface heat fluxes. A method for using microwave brightness temperature observation to estimate $Q_{a}$ and $T_{a}$ has been presented. The innovative aspect of this study is the ANN approach with satellite observed microwave brightness temperature.

The evaluation of the method shows good agreement with the surface marine observation not used in the development of algorithm. The global rms error in $Q_{a}$ is $1.1 \mathrm{~g} / \mathrm{kg}$. Likewise, the global rms 

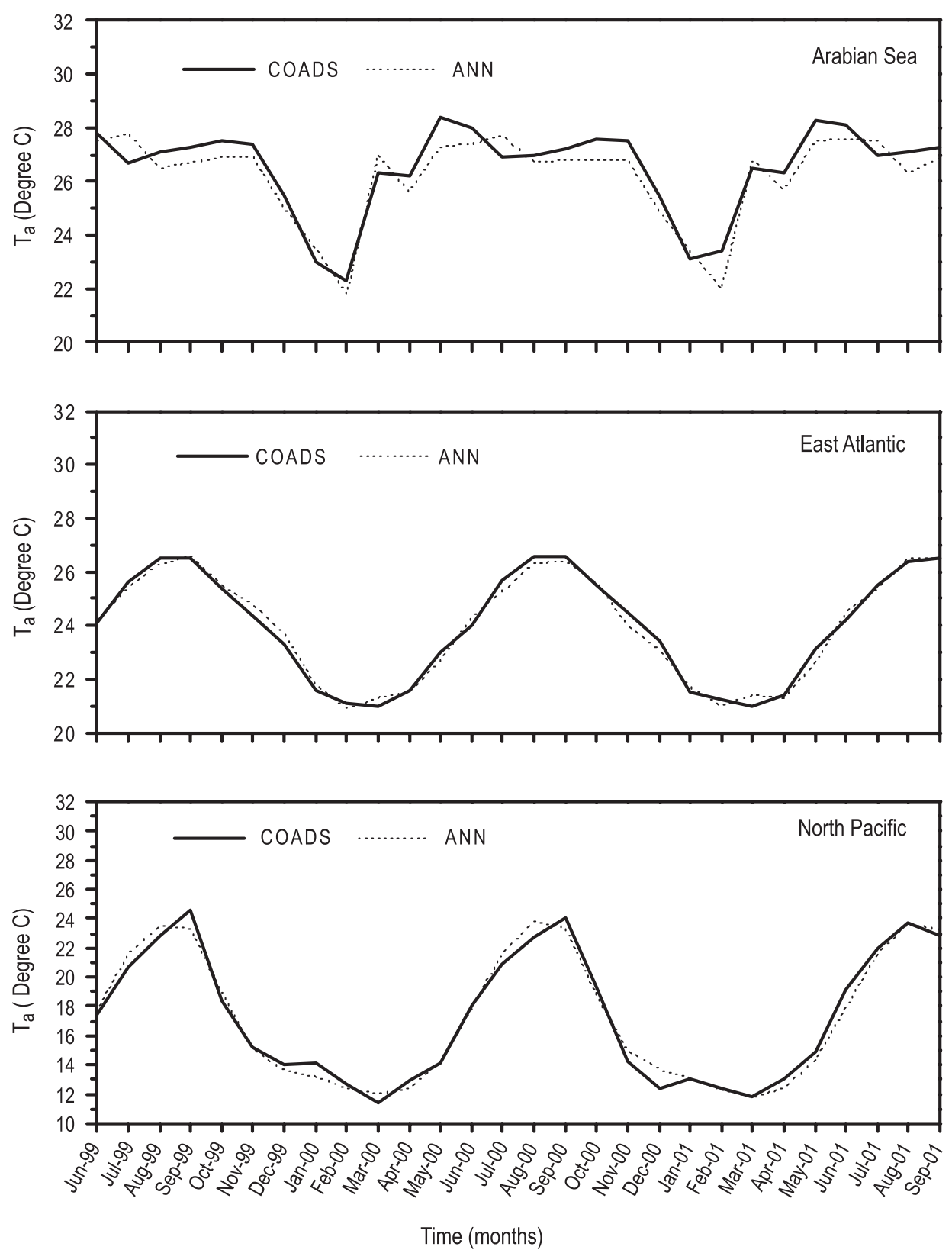

Figure 10. Same as figure 9 but for air temperature $\left({ }^{\circ} \mathrm{C}\right)$.

error in $T_{a}$ is $1.0^{\circ} \mathrm{C}$. In the region of high ship observations (north Pacific and north Atlantic Oceans) rms differences in $T_{a}$ and $Q_{a}$ are about $0.6 \mathrm{~g} / \mathrm{kg}$ and $0.7^{\circ} \mathrm{C}$. The differences between COADS and satellite retrieved parameters $\left(Q_{a}\right.$ and $\left.T_{a}\right)$ are more over the southern hemisphere. The cause of the differences is very difficult to judge. Because the ship observations are very less over these regions.

The space-borne retrieval of wind speed, sea surface temperature along with near surface specific humidity and surface air temperature, will improve the latent and sensible heat fluxes derived from satellite measurements. The MSMR estimated surface specific humidity and surface air temperature in turn could be used for applications in atmospheric numerical models.

From the results, one may conclude that brightness temperature from satellite such as MSMR may provide reasonable estimates of surface specific humidity and air temperature over oceanic regions. This method could be useful for climate modeling by providing consistent and independent estimates of global $Q_{a}$ and $T_{a}$. The results also further demonstrate the potential of microwave radiometers. 


\section{Acknowledgement}

We thank Climate Data Centre for making the COADS data available on their site ftp://ftp.cdc.noaa.gov. Authors are thankful to Dr. M S Narayanan, Dr. Abhijit Sarkar and Dr. B S Gohil of the Meteorology \& Oceanography Group, for fruitful discussions.

\section{References}

Esbensen S K, Chelton D B, Vockers D and Sun J 1993 An analysis of errors in Spatial Sensor Microwave Imager evaporation estimates over the global oceans; J. Geophys. Res. 96 7081-7101

Fletcher J O, Slutz R J and Woodruff S D 1983 Towards a comprehensive ocean atmosphere data set, trop; Ocean Atmos, News Lett. 20 13-14

Fausett L 1994 Fundamentals of neural network: Architecture, algorithms and applications (New Jersey: Prentice Hall)

Gohil B S, Mathur A K and Varma A K 2000 Geophysical parameters retrieval over global ocean from IRSP4/MSMR, PORSEC Symp., Goa, India

Jourdan D and Gautier C 1995 Comparison between global latent heat flux computed from multisensor (SSM/I, AVHRR) and from in situ data; J. Atmos. Oceanic Technol. 12 46-72
Jones C, Peterso P and Gautier C 1999 A new method for deriving ocean surface specific humidity and air temperature: An artificial neural network approach; J. App. Meteorol. 38 1229-1245

Jung T, Ruprecht E and Wagner F 1998 Determination of cloud liquid water path over oceans from special sensors microwave / imager (SSM/I) data using neural networks; J. App. Meteorol. 37 832-844

Konda M, Imasato N and Shibata A 1996 A new method to determine near-surface air temperature by using satellite data; J. Geophys. Res. 101 14349-14360

Liu W T 1986 Statistical relation between monthly mean precipitable water and surface-level humidity over global oceans; Mon. Weather Rev. 114 1591-1602

Liu W T 1988 Moisture and latent heat fluxes variabilities on the tropical pacific derived from satellite data; J. Geophys. Res. 93 6749-6760

Miller S W and Emery W J 1997 An automated neural network and cloud classifier for use over land and ocean surfaces; J. Appl. Meteorol. 36 1346-1362

Oort A H, Pan Y H, Reynolds R W and Popelewski C F 1987 Historical trends in surface temperature over the oceans based on COADS; Climate Dyn. 2 29-36

Peixoto J P and Oort A H 1992 Physics of Climate; American Institute of Physics, 520 pp.

Rumelhart D E, Hinton G and Williams R 1986 Learning representations by error propagation to adaptive learning algorithms: Foundations; D E Rumelhart and J L Mc C (eds) (The MIT Press) 318-362

Schulz J, Meywerk J, Edward S and Schlussel P 1997 Evaluation of satellite derived latent heat fluxes; J. Climate. $102782-2795$ 